\title{
Synthesis and Characterization of Nano-Acetamiprid-New Plant Safeguard Nanomaterial
}

\author{
P. Padmavathi, N. Vasundhara, Swathi Kovvuri, N. V. S. Venugopal* \\ Department of Chemistry, Institute of Science, GITAM University, Visakhapatnam, India \\ Email: *venu7000@gmail.com
}

How to cite this paper: Padmavathi, P., Vasundhara, N., Kovvuri, S. and Venugopal, N.V.S. (2020) Synthesis and Characterization of Nano-Acetamiprid-New Plant Safeguard Nanomaterial. American Journal of Analytical Chemistry, 11, 197-204. https://doi.org/10.4236/ajac.2020.115015

Received: March 30, 2020

Accepted: April 25, 2020

Published: April 28, 2020

Copyright (C) 2020 by author(s) and Scientific Research Publishing Inc. This work is licensed under the Creative Commons Attribution International License (CC BY 4.0).

http://creativecommons.org/licenses/by/4.0/

\begin{abstract}
In present days many types of materials are used to reduce the environmental pollution in the world which includes nanomaterials. Nanopesticides increase the efficacy, durability and reduction in the amount of active constituent. The potential applications of nanotechnology in pesticides are quick decomposition in soil or plant, targeted delivery, apparent solubility and controlled release. In this communication the author reported a neonicatonoid insecticide called as Nano-acetamiprid and it is widely used to control fungal infections in different crops like cotton, leafy vegetables, citrus fruits, pome etc. The author reported a facile method i.e. a new Nano-acetamiprid for plant disease control and its subsequent characterization of encapsulated complex using polycaprolactone as an encapsulated agent. Nano-acetamiprid encapsulated particles were characterized by dynamic light scattering (DLS), ultraviolet spectroscopy and scanning electron microscopy (SEM). To ascertain the formation and the stability of nanoencapsulated acetamiprid pesticide, the maximum absorption spectra formulated at $421 \mathrm{~nm}$ and unformulated pesticide at $520 \mathrm{~nm}$ were observed. The size distribution was noted at $40-50 \mathrm{~nm}$. The bioactivity study was conducted against various Aspergillus niger. The performance of nano particles was many fold times effective when compared to the original parental particles. The bio-assay of Nano-acetamiprid shows better results when compared to the normal commercial acetamiprid.
\end{abstract}

\section{Keywords}

Acetamiprid, Nano Encapsulation, Polycaprolactone, Antifungal Studies

\section{Introduction}

Acetamiprid is a neonicotinoid with a chloropyridinyl group and it is used to 
protect plants against sucking aphid insects. It is also applied on plants ranging from leafy vegetables and fruit trees to ornamental plants. Acetamiprid is a carboxamidine that is acetamidine in which the amino hydrogens are substituted by a (6-chloropyridin-3-yl) methyl and a methyl group while the hydrogen attached to the imino nitrogen is replaced by a cyano group. Acetamiprid is an organic compound with the chemical formula $\mathrm{C}_{10} \mathrm{H}_{11} \mathrm{ClN}_{4}$. (molar mass $222.67 \mathrm{~g} / \mathrm{mol}$ ). It is an odorless neonicotinoid insecticide produced. It has a role as a neonicotinoid insecticide, an environmental contaminant and a xenobiotic. It is a monochloropyridine, a nitrile and a carboxamidine. It derives from a 2-chloropyridine. Acetamiprid has a high potential for bioaccumulation and is highly toxic to birds and moderately toxic to aquatic organisms. Excessive use of the pesticide could pose a threat to human life.

Researchers concentrated on recent development of nanotechnology [1]. New colorimetric sensors based on the aggregation of metallic nanoparticles are emerging [2] [3] [4] [5]. Among all the materials explored, gold nanoparticles (AuNPs) have received more consideration owing to their intrinsic characteristics such as easy preparation, biocompatibility, stability, and high extinction coefficients [6]. Various substances have been detected by the gold nanoparticles based colorimetric method [7]-[12]. Encapsulation process has wide applications such as foods, cosmetics, paints, paper, textiles etc. To remove harmful effects on the non-target organisms, encapsulation of the active ingredient with other materials such as a polymer can allow sensitive ingredients to be physically enveloped into a protective matrix in order to protect core materials from adverse reactions due to factors like air or light. Rapid growth has been found in the field of nanotechnology over the past few years and it has successfully ventured into different fields of science. Nanoformulations of pesticides based on polymers have been exploited for the encapsulation of most of the insecticides. The first polymer contained formulation was developed in the year 1970 for control release of biocides. Techniques like electrochemical synthesis, chemical vapour deposition, sol-gel synthesis, microwave assisted synthesis, ultrasonication, "precipitation method etc", were reported for the synthesis of nanoparticles [13] [14] [15]. How nanoparticles can act and control of pests is less studied. In certain cases pesticide nanoparticles present possibilities for controlling pest effectively [16] [17]. Few studies reported on polymer nanocomposites like polyethylene glycol due to its properties and multifunctional structures of each segment [18] [19].

Hence the nano size of pesticide particles could reduce the threat to the living organisms. In this communication we reported a non-hazardous polycaprolactone as a capping agent to prepare the nano formulation of acetamiprid pesticide. Ever since nanopesticides emerge as a developing field in agriculture for best yields, better penetration towards the target molecule, low concentration of pesticide moleculs. Increased surface area of nano shaped particles allows us to use the less quantity of traditional pesticides in the agricultural land. 


\section{Materials and Methods}

\subsection{Reagents}

Polycaprolactone was procured from E. Merck, India. All other chemicals used were of analytical grade. Water used throughout the process was double-distilled water.

Acetamiprid was generously gifted by S. N. Agro traders, Visakhapatanam, India. Glassware used in this experimental work was properlyacid washed.

\subsection{Preparation of Nano-Acetamiprid}

Commercially available acetamiprid is finely grounded with the help of mortar and pestle. The soluble form of the finely powdered acetamiprid was mixed in ultra sonicate bath for 40 minutes with the twice the polycaprolactone solvent. The formulation was subjected to continuous mixing followed by stirring for 5 6 hours and then the excess solvent was removed by using Rota-evaporator.

\subsection{Instrumentation}

Particle size distribution was obtained by Dynamic light scattering (DLS) Horiba, nanopartica, Japan, s2100. UV-V is measurements were obtained by using Shimadzu UV-1650 PC Spectrophotometer through a quartz cell with $10 \mathrm{~mm}$ optical path and this was used to characterize and identify the organic molecule. Surface topology was determined through scanning electron microscopy (Zeiss Evol8).

\section{Results and Discussion}

The pressing concern of the world at present is the excessive use of agrochemicals and it leads to risks for human health and many environmental problems. So many observations foresee that nanoscience and nanotechnology allow the development of agricultural fields with high technology contains nanoproducts and that leads precise management and less utilization of pesticides. Nanopesticides are the best alternative to the traditional pesticides.

Nano-acetamiprid is highly active at lower level concentration and reduces toxicity in agricultural fields. The polycaprolactone enhances the stability and it slowly releases the active ingredient to the target. The mode of action of Nano-acetamiprid is shown in Figure 1.

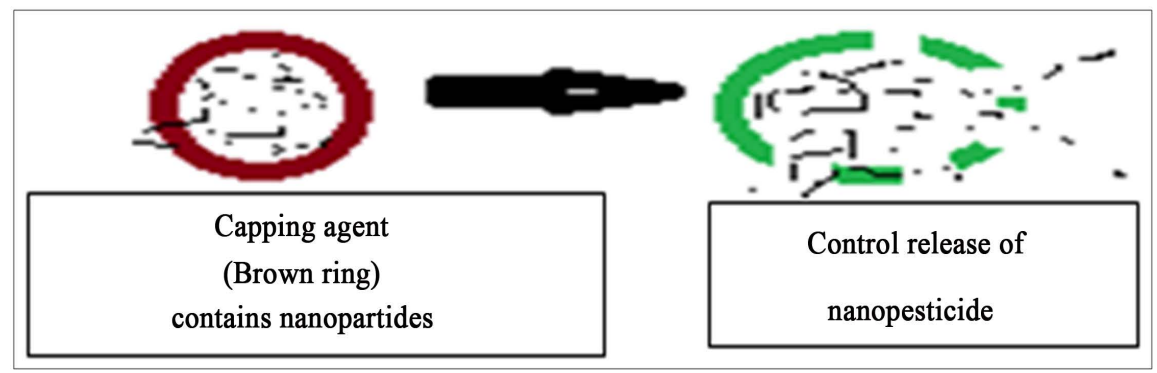

Figure 1. Mode of action of Nano-acetamiprid. 
Nano-acetamaprid reduces toxicity in agricultural fields and it is highly active at lower level concentration. In general better efficacy, better control of application and less use of chemicals is the benefits of nanopesticides. Our study provides a novel nanoformulation of acetamiprid pesticide by Polycaprolactone as capping agent. The polycaprolactone enhances the stability and it slowly releases the active ingredient to the target. The structure of acetamiprid and polycaprolactone (capping agent) are shown in Figure 2 and Figure 3.

\subsection{DLS Analysis}

The diameter of the nano-encapsulated acetamiprid was obtained by pade laplace dispersion. It mainly depends on Rayleigh scattering, oscillations, Brownian movement and fluorescence exponential decay. In this method a time dependent signal was transformed into the hydro colloidal solution and it results in the exponential decay of the particles. The transformed laplace signal is defined as an infinite integral over time. Through this transformed signal number of components in the encapsulated solution can be easily obtained. It can be clearly depicted in the following pade laplace dispersion graph. Dynamic light scattering (DLS) measures the scattering intensity based on Rayleigh scattering. One milli litre of nano-encapsulated acetamiprid was suspended in $5 \mathrm{ml}$ of water. The resultant hydro dispersed suspension was analyzed with DLS at $25^{\circ} \mathrm{C}-40^{\circ} \mathrm{C}$. The particle size distribution was appeared around $40-50 \mathrm{~nm}$. The particle size distribution is shown in Figure 4.



Figure 2. Structure of acetamiprid.

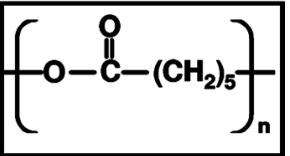

Figure 3. Structure of polycaprolactone (capping agent).

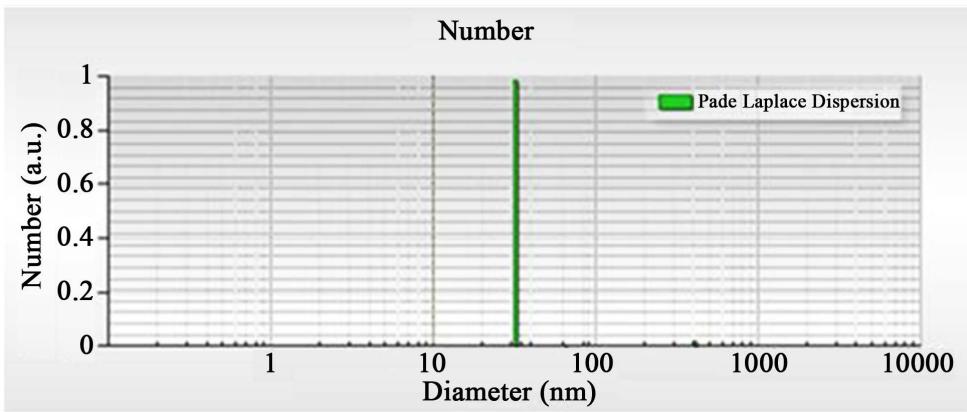

Figure 4. Nano-acetamiprid size distribution. 


\subsection{UV-Vis Spectral Study}

To ascertain the formation and the stability of nanoencapsulated acetamiprid pesticide, UV-Vis spectra is necessary. 19 The maximum absorption spectra formulated at $421 \mathrm{~nm}$ and unformulated pesticide at $520 \mathrm{~nm}$ were shown in Figure 5.

\subsection{SEM Analysis}

The surface topology, size of Nano-acetamiprid was obtained by scanning electron microscope at $500 \times-35 \mathrm{k} \times$ magnification. A drop of encapsulation complex is taken on the stub and it was air dried then subjected to sputtering using sputter coater. The SEM image reveals the homogeneity in shape and it is regular among different scan a regions. SEM image which was depicted in Figure 6 shows a number of Nano-acetamiprid particles in the clusters.

\subsection{Application of Nano Acetaprid on Aspergillus bacillus}

The antifungal activity of encapsulated acetamiprid was examined in petridish assay by disc diffusion method. The fungal isolates were cultured on potato dextrose agar (PDA) medium which induce the conidia production incubated at $37^{\circ} \mathrm{C}$ for 10 days. Sterile de-ionized water was used as control. The antifungal activity of encapsulated acetamiprid was examined in petridish assay by disc diffusion method [20]. The fungal isolates were cultured on potato dextrose (PDA) medium which induce the conidia for 10 days. Sterile ionized water was used as control. The sample ppm, $40 \mathrm{ppm}$ and $20 \mathrm{ppm}$, diluted 100-fold with de-ionized water. On to the PDA mediums filter paper discs dipped in different ppm were inserted and the petridishes were incubated at $37^{\circ} \mathrm{C}$ for $2-4$ days respectively. The size of the zone was determined by measuring the diameter of the zone in $\mathrm{mm}$. Nano encapsulated acetamiprid shows best results when compared with commercial acetamiprid which can be clearly depicted below. The various inhibitory zones of Nano-acetamiprid samples are shown in Figure 7.

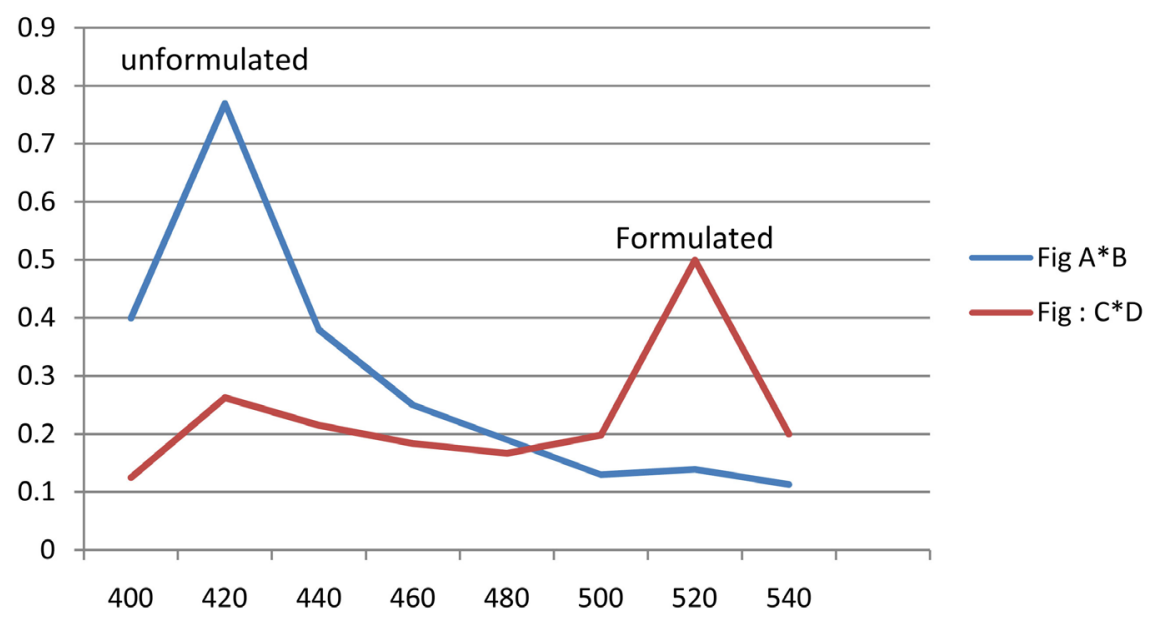

Figure 5. Maximum absorption of formulated and unformulated acetamiprid. 


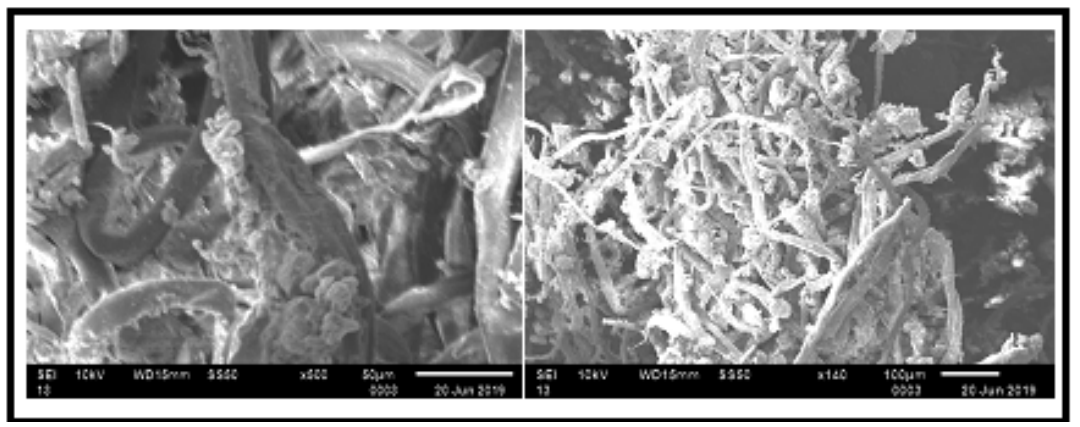

Figure 6. SEM images of Nano-acetamiprid.

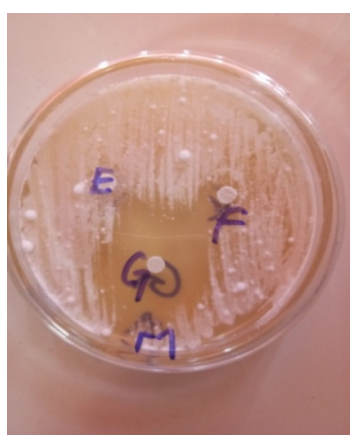

(a)

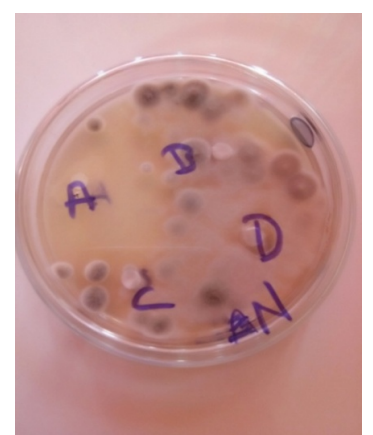

(b)

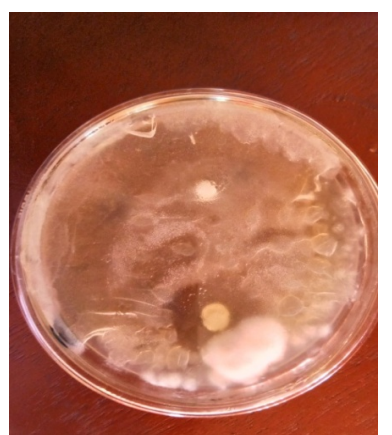

(c)

Figure 7. Inhibitory zones ((a), (b), and (c)) of Aspergillus niger. (a) Nano-acetamiprid on agar plates; (b) Formation of clear zone; (c) Nano-acetamiprid inhibitory zone at 50 ppm.

\section{Conclusion}

Nanoscience has greatly contributed to major achievements in various fields. The biodegradable additive polycaprolactone is used to minimize the size of pesticide molecules. A better alternative to conventional pesticide is nanopesticide. Nano-acetamiprid was formulated by using polycaprolactone as capping agent. The properties of characterization of different techniques support the encapsulation, characterization and stability of Nano-acetamiprid pesticide. The nano encapsulated acetamiprid was found to have more antifungal activity. The result suggests that the synthesized nano-carbendazim pesticide undergo interaction with bacterial cell and thereby exhibit strong action. The formation of clear zone with restricted bacterial growth around the cavity is shown in the figure. The valuable information has paved way to further development and practical application of polymeric nano insecticides with huge potential.

\section{Acknowledgements}

We are deliberately thankful to the department of chemistry, Institute of science, GITAM University for their immense support and huge encouragement.

\section{Conflicts of Interest}

The authors declare no conflicts of interest regarding the publication of this re- 
search paper.

\section{References}

[1] Liu, S.Q., Yuan, L., Yue, X.L., Zhang, Z.Z. and Tang, Z.Y. (2008) Recent Advances in Nanosensors for Organophosphate Pesticide Detection. Advanced Powder Technology, 19, 419-441. https://doi.org/10.1016/S0921-8831(08)60910-3

[2] Liu, J.W. and Lu, Y. (2006) Colorimetric Sensing of Adenosine and Cocaine Based on a General Sensor Design Involving Aptamers and Nanoparticles. Angewandte Chemie International Edition, 45, 90-94. https://doi.org/10.1002/anie.200502589

[3] Wu, W.Y., Bian, Z.P., Wang, W. and Zhu, J. (2010) Gold Nanoparticle Composite Film-Based Silver Enhanced Colorimetric Detection of Cardiac Troponin. Sensors and Actuators B: Chemical, 147, 298-303. https://doi.org/10.1016/j.snb.2010.03.027

[4] Zhang, Y.M., Lin, Q., Wei, T.B., Wang, D.D., Yao, H. and Wang, Y.L. (2009) Simple Colorimetric Sensors with High Selectivity for Acetate and Chloride in Aqueous Solution. Sensors and Actuators B: Chemical, 137, 447-455.

https://doi.org/10.1016/j.snb.2009.01.015

[5] Zhang, S.H., Wang, J., Han, L., Li, C.G., Wang, W. and Yuan, Z. (2010) Colorimetric Detection of Bis-Phosphorylated Peptides Using Zinc (II) Dipicolylamine-Appended Gold Nanoparticles. Sensors and Actuators B: Chemical, 147, 687-690. https://doi.org/10.1016/j.snb.2010.03.071

[6] Rosi, N. and Mirkin, C.A. (2005) Nanostructures in Biodiagnostics. Chemical Reviews, 105, 1547-1562. https://doi.org/10.1021/cr030067f

[7] Ai, K.I., Liu, Y.L. and Lu, L.H. (2009) Hydrogen-Bonding Recognition Induced Color Change of Gold Nanoparticles for Visual Detection of Melamine in Raw Milk and Infant Formula. Journal of the American Chemical Society, 131, 9496-9497. https://doi.org/10.1021/ja9037017

[8] Han, C.P., Zeng, L.L., Li, H.B. and Xie, G.Y. (2009) Colorimetric Detection of Pollutant Aromatic Amines Isomers with p-Sulfonatocalix Arene-Modified Gold Nanoparticles. Sensors and Actuators B: Chemical, 137, 704-709. https://doi.org/10.1016/j.snb.2008.12.038

[9] Zhao, W., Brook, M.A. and Li, Y.F. (2008) Design of Gold Nanoparticle Based Colorimetric Biosensing Assays. ChemBioChem, 9, 2363-2371.

https://doi.org/10.1002/cbic.200800282

[10] Zhao, W., Chiuman, J.C.F., Lam, S.A., McManus, W., Chen, Y.G., Cui, R., Pelton, M.A., Brook, A. and Li, Y.F. (2008) DNA Aptamer Folding on Gold Nanoparticles: From Colloid Chemistry to Biosensors. Journal of the American Chemical Society, 130, 3610-3618. https://doi.org/10.1021/ja710241b

[11] Zhao, W., Chiuman, W., Brook, M.A. and Li, Y.F. (2007) Simple and Rapid Colorimetric Biosensors Based on DNA Aptamer and Noncrosslinking Gold Nanoparticle Aggregation. ChemBioChem, 8, 727-731. https://doi.org/10.1002/cbic.200700014

[12] Zhang, Y.F., Li, B.X. and Chen, X.L. (2010) Simple and Sensitive Detection of Dopamine in the Presence of High Concentration of Ascorbic Acid Using Gold Nanoparticles as Colorimetric Probes. Microchimica Acta, 168, 107-113. https://doi.org/10.1007/s00604-009-0269-5

[13] Horwat, D.I., Zakharov, J.L., Endrino, et al. (2011) Chemistry, Phase Formation, and Catalytic Activity of Thin Palladium-Containing Oxide Films Synthesized by Plasma-Assisted Physical Vapor Deposition. Surface and Coatings Technology, 205, 
S171-S177. https://doi.org/10.1016/j.surfcoat.2010.12.021

[14] Chandraboss, V.L., Senthilvelan, S., Natanapatham, L.M., Murugavelu, B., Loganathan, B. and Karthikeyan, J. (2013) Photocatalytic Effect of Ag and Ag/Pt Doped Silicate Non Crystalline Material on Methyl Violet-Experimental and Theoretical Studies. Journal of Non-Crystalline Solids, 368, 23-28.

https://doi.org/10.1016/j.jnoncrysol.2013.02.027

[15] Karthikeyan, B. and Loganathan, B. (2013) Rapid Green Synthetic Protocol for Novel Trimetallic Nanoparticles. Journal of Nanoparticles, 2013, Article ID: 168916. https://doi.org/10.1155/2013/168916

[16] Karunaratne, V., Kottegoda, N. and Alwis, A.J. (2012) National Science Foundation, 40, 3-8. https://doi.org/10.4038/jnsfsr.v40i1.4165

[17] Khot, L.R., Sankaran, S.H., Maja, E.W., et al. (2012) Applications of Nanomaterials in Agricultural Production and Crop Protection: A Review. Crop Protection, 35, 64-70. https://doi.org/10.1016/j.cropro.2012.01.007

[18] Ajayan, P.M. (1999) Nano Tubes from Carbon. Chemical Reviews, 99, 1787-1799. https://doi.org/10.1021/cr970102g

[19] Choudary, S.R., Pradhan, S. and Goswami, A. (2012) A Preparation and Characterisation of Acephate Nanocomplex. Nanoscience Methods, 1, 9-15. https://doi.org/10.1080/17458080.2010.533443

[20] Barry, A.L. and Brown, S.D. (1996) Fluconazole Disk Diffusion Procedure for Determining Susceptibility of Candida Species. Journal of Clinical Microbiology, 34, 2154-2157. https://doi.org/10.1128/JCM.34.9.2154-2157.1996 